\title{
Editorial
}

\section{Very exotic derivatives}

Journal of Derivatives \& Hedge Funds (2013) 19, 243. doi:10.1057/jdhf.2014.7

Our special issue concerns exotic derivative. A definition of an exotic derivative seems to be a derivative that is not plain vanilla. It is a pleasure to present a number of papers that address pricing issues for exotic derivatives. It is a topic that I would have liked to present a special issue on but, up until now, have not had suitable material.

The publications in this issue are indeed a great distance from plain vanilla; one example, by Geng Deng and co-authors, looks at mis-pricing in reverse convertibles, which 'embed downand-in put options and offer investors relatively high coupon payments in exchange for bearing some of the downside risk of the equity underlying the note'. The analysis is based on the Variance-Gamma model and the authors find evidence of over-pricing. As these are retail products, perhaps over-pricing reflects the restricted access to non-linear payoffs that most retail investors experience.

I will not go through a paper-by-paper scrutiny of the contents of this special issue but I note another paper by Tim Xiao, who presents a new framework for valuing hybrid defaultable financial instruments, for example, convertible bonds. In contrast to previous studies, the model relies on the probability distribution of a default jump, and the author uses this to back out prices from this distribution. Again, the theme is mis-pricing.

There is a deeper philosophical issue behind this literature which is that we know what the fair price is. This is one of those issues that becomes more complicated the longer one thinks about it. The gist of the problem is whether we know what the true price of something is. Surprisingly, Karl Marx addressed this with his Labour theory of Value; the true price being measured in hours of labour time directly and indirectly embedded in the commodity. Such an approach is a long way from finance where we start with some prices being assumed to be true, equity prices perhaps for plain vanilla equity options and then we infer the true price of the derivative because the derivative pay-off is a function of the equity pay-off. This gets much harder the more exotic the derivative as we can see if we were to consider house-price options, weather options and so on. None of these pessimistic thoughts should reduce in any way the interest readers will find in the papers in this issue.

Steven Satchell 